\title{
Descripción clínico-epidemiológica y molecular del cáncer de pulmón en un centro de referencia nacional
}

\author{
Clinical-epidemiological and molecular description of \\ lung cancer in a national reference center
}

\author{
Carla Paola Sánchez-Ríos, ${ }^{*}$ Jerónimo Rafael Rodríguez-Cid, ${ }^{*}$ Luis Manuel Martínez-Barrera,* \\ Patricio Santillán-Doherty, Jorge Arturo Alatorre-Alexander*
}

*Instituto Nacional de Enfermedades Respiratorias Ismael Cosío Villegas, Ciudad de México, México.

\begin{abstract}
RESUMEN. Introducción: El cáncer de pulmón es la primera causa de muerte por cáncer a nivel mundial, muestra un incremento en mujeres y en no fumadores en la última década. El progreso en el abordaje diagnósticoterapéutico se ha logrado gracias al conocimiento de la heterogeneidad tumoral y al análisis molecular. Material y métodos: Se analizó la base de pacientes con cáncer de pulmón de enero de 2013 a junio de 2018, con registro de variables sociodemográficas, clínicas y perfil molecular. Resultados expresados mediante medidas de tendencia central y se estimó el odd ratio crudo para mutación de receptor del factor de crecimiento epidérmico y antecedente exposicional y su significancia estadística mediante prueba exacta de Fisher. Resultados: Se analizó una cohorte con un total de 751 pacientes. La edad promedio fue de 62.2 años, $53 \%(n=398)$ fueron del género femenino, $51.8 \%(\mathrm{n}=389)$ nunca fueron fumadores. Los síntomas más comunes al diagnóstico fueron tos en $87.1 \%$ y disnea en $74.4 \%$. El procedimiento broncoscópico fue el método diagnóstico preferido. El estadío clínico IV fue el más frecuentemente reportado. Se encontró 38.19\% de cáncer de pulmón de células no pequeñas con receptor del factor de crecimiento epidérmico positivo. Conclusión: La situación clínico-epidemiológica del Instituto Nacional de Enfermedades Respiratorias Ismael Cosío Villegas se asemeja a las estadísticas mundiales en diagnóstico y tratamientos.
\end{abstract}

\begin{abstract}
Introduction: Lung cancer is the leading cause of cancer death worldwide, showing an increase in women and non-smokers in the last decade. Progress in the diagnostic-therapeutic approach has been achieved thanks to the knowledge of tumor heterogeneity and molecular analysis. Material and methods: The base of patients with lung cancer was analyzed from January 2013 to June 2018, with registration of sociodemographic, clinical and molecular profile variables. Results expressed through measures of central tendency and crude odd ratio was estimated for EGFR mutation and exposure antecedent and its statistical significance by Fisher's exact test. Results: A cohort analyzed from January 2013 to June 2018 with 751 patients. The average age was 62.2 years, $53 \%(n=398)$ were female, $51.8 \%(n=389)$ were never smokers. The most common symptoms were cough in $87.1 \%$ and dyspnea in $74.4 \%$. The bronchoscopic procedure was the preferred diagnostic method. Clinical stage IV was the most frequently reported. EGFR was positive in $38.19 \%$ of NSCLC. Conclusion: The clinical-epidemiological situation of the INER resembles global statistics in both diagnosis and personalized treatments.
\end{abstract}

Keywords: Lung cancer, non-smoking, prognosis.

Palabras clave: Cáncer pulmonar, no fumadores, pronóstico.

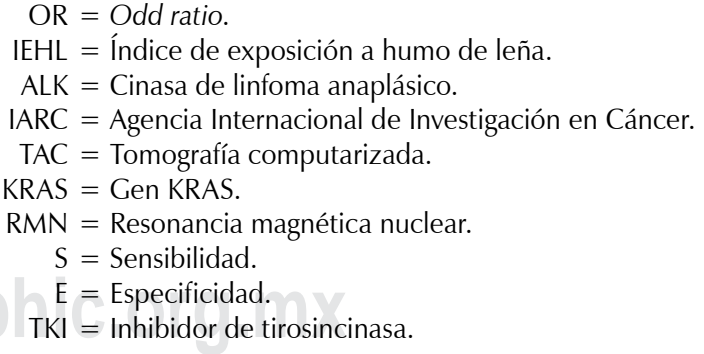

Correspondencia:

Dr. Jorge Arturo Alatorre Alexander

Instituto Nacional de Enfermedades Respiratorias Ismael Cosío Villegas. Ciudad de México, México. Correo electrónico: dr.j.alatorresq@gmail.com

Trabajo recibido: 12-III-2019. Aceptado: 16-V-2019. 


\section{INTRODUCCIÓN}

El cáncer de pulmón (CP) es un problema de salud pública. ${ }^{1}$ En los inicios de la epidemia del CP más de 90\% de los casos se atribuían al tabaquismo; sin embargo, en las últimas décadas esta proporción ha evidenciado un cambio importante. En la actualidad se estima que $20-25 \%$ de los casos de CP ocurren en sujetos nunca fumadores, en quienes se considera una etiología multifactorial dentro de la cual se han investigado diversos carcinógenos ambientales, ocupacionales y factores genéticos. ${ }^{2-5}$

El tabaquismo como causa de CP ha sido ampliamente estudiado y está bien establecido que a menor edad de inicio, a mayor cantidad de cigarrillos y años fumando, así como la predisposición genética, es mayor el riesgo de desarrollar la enfermedad. El IT es la medida más objetiva utilizada de la intensidad de exposición al humo del tabaco por una persona. Entre mayor es el IT, mayor es el riesgo de desarrollar CP, y el riesgo es más evidente a un IT a partir de 20 paquetes/año. La contaminación ambiental producida por combustión incompleta de combustibles fósiles parece incrementar el riesgo de desarrollar CP, y la exposición a aeropartículas del humo de leña tiene un impacto importante como factor de riesgo de carcinogénesis pulmonar y de otras patologías pulmonares.

Una forma de dar objetividad a la exposición ambiental a biomasa es con el índice de exposición al humo de leña y aunque para el CP no se ha determinado a partir de qué nivel de corte aumenta el riesgo, se considera que si sobrepasa las 200 h/año, éste aumenta. ${ }^{6}$ Otros carcinógenos ambientales son el radón, el asbesto y el arsénico. ${ }^{7}$ En la actualidad se diferencia entre dos tipos de CP según el crecimiento celular tumoral, el tratamiento y el pronóstico:

1. El cáncer pulmonar de celulas pequeñas (СРCP) representa 20\% de todos los casos de CP. Esta forma de cáncer tiene un crecimiento más rápido, es de curso más agresivo y produce metástasis con relativa rapidez. Debido a ello, el pronóstico es peor comparado con el cáncer de pulmón de células no pequeñas (CPCNP). En alrededor de $80 \%$ de las personas con CPCP, la enfermedad se diagnostica en etapas avanzadas.

2. CPCNP se subdivide a su vez en: adenocarcinoma, que representa el subtipo histológico predominante $(60 \%$ de todos los tipos de CP) y sobre el cual se han realizado avances significativos en términos de tratamiento en los últimos años gracias a la caracterización de paneles moleculares utilizados como blancos terapéuticos, tal es el caso de las mutaciones en el gen del EGFR. Un problema mayor con esta entidad es la frecuente heterogeneidad histológica encontrada, lo cual impacta en el pronóstico. El carcinoma de células escamosas, otro subtipo de CPCNP ubicado en la mayoría de los casos cerca de los bronquios principales, es el CPCNP más asociado a tabaquismo. ${ }^{8-10}$
Con respecto a los estadíos de la enfermedad, la estadificación hoy se basa en la clasificación de TNM octava edición.

Como problema de salud pública, el CP causa altos costos económicos, tanto para instituciones del sector salud como del sector privado, con un costo anual promedio por paciente mayor de 100 mil pesos en algunos centros. ${ }^{11}$ Este panorama es debido en parte a la incapacidad de los servicios de salud para ofrecer un diagnóstico y tratamiento oportuno que conlleva largas estancias intrahospitalarias de los pacientes; y en la mayoría de los casos deterioro clínico por la presencia de comorbilidades asociadas con la enfermedad o con el tratamiento a base de citotóxicos. Se sabe que un paciente diagnosticado en estadíos clínicos tempranos posee mayor índice de respuesta al tratamiento, cercano a $70 \%$. En contraste, sólo $15 \%$ de los pacientes responden de manera parcial en estadíos avanzados con un bajo índice de sobrevida. ${ }^{12}$

En México más de $80 \%$ de los pacientes que son diagnosticados con esta enfermedad se detectan en etapa avanzada. Los nuevos tratamientos basados en paneles moleculares, aunque han sido mejor estudiados en etapas avanzadas de la enfermedad, muestran beneficio claro en aumento de SLP y SG comparados con quimioterapia estándar basada en platinos. Sin embargo, las complicaciones asociadas como obstrucción vía aérea central, derrame pleural maligno sintomático, hemoptisis, entre otras, contribuyen en el deterioro del estado funcional de los pacientes a corto plazo, lo cual impacta en la oportunidad de poder ofrecer tratamientos sistémicos y en el pronóstico. ${ }^{13-16}$

El INER Ismael Cosío Villegas, Ciudad de México, ofrece la identificación de mutaciones en gen de EGFR por análisis de PCR automatizado y aunque en su momento fue uno de los grandes avances en materia de oncología, hoy sabemos que no es la única mutación genética que puede usarse como diana terapéutica. Los avances en relación al tratamiento de CP basados en la búsqueda e identificación de mutaciones somáticas por técnicas de NSG aunado al trabajo sumado de médicos oncólogos, investigadores, biólogos moleculares y neumólogos con expertiz en $\mathrm{CP}$ del instituto ha generado un aumento en la accesibilidad a estas herramientas permitiendo en gran medida cronificar la enfermedad.

El objetivo general de este trabajo fue describir cuál es el perfil clínico epidemiológico del CP de pacientes atendidos en el INER Ismael Cosío Villegas. Asimismo, se describieron las prevalencias de CP por género y factor de exposición, prevalencias de CP por histología, prevalencias de CPCNP no escamoso por patrones histológicos, prevalencia de mutaciones tipo EGFR en pacientes con adenocarcinoma pulmonar y los porcentajes de pacientes por línea de tratamiento implementada y porcentaje de aquéllos que recibieron algún tipo de inmunoterapia para $\mathrm{CP}$. 


\section{MATERIAL Y MÉTODOS}

Se trata de un estudio observacional, retrospectivo y transversal realizado en el INER Ismael Cosío Villegas, Ciudad de México, México.

Se reclutaron expedientes correspondientes a la población del INER con diagnóstico de CP de enero de 2013 a junio de 2018, cuyos datos se determinaron de manera retrospectiva por parte de los investigadores principales con el llenado de hojas de cotejo formuladas por los mismos para determinación del perfil clínico y epidemiológico del $\mathrm{CP}$, registrándose las siguientes variables: género, edad, estado de procedencia, sintomatología, toma de muestra, resultado histopatológico, estudios de extensión para estadificación, panel molecular y tratamiento. Los resultados fueron analizados y expresados mediante medidas de tendencia central y se estimó el odd ratio crudo para mutación EGFR y antecedente de tabaquismo con su significancia estadística mediante prueba exacta de Fisher.

\section{RESULTADOS}

Se analizó una cohorte de enero de 2013 a junio de 2018 con un total de 751 pacientes con diagnóstico de CP en el INER Ismael Cosío Villegas. La edad promedio al momento del diagnóstico fue de 62.2 años. Del total de pacientes, $53 \%(n=398)$ fueron del género femenino. Las principales localidades de las que procedían los pacientes al momento del diagnóstico fueron en orden descendente: Ciudad de México 37.6\% $(\mathrm{n}=279)$, Estado de México 28.4\% ( $\mathrm{n}=$ 211) y Guerrero $5.1 \%(n=38)$.

Se registraron los antecedentes exposicionales de los pacientes en cuatro rubros principales: tabaquismo, humo de leña, asbesto e hidrocarburos. Respecto al antecedente de tabaquismo que se encontró en $48.2 \%(n=362)$, se estratificó en dos grupos: fumador actual $31.4 \%(n=114)$ y exfumador $68.6 \%(n=248)$. El grupo de nunca fumadores representó $51.8 \%(n=389)$ del total de la cohorte. De los pacientes considerados fumadores, $28.1 \%$ tenían antecedente de exposición significativo con IT por arriba de 20 paquetes al año. Respecto a la exposición de biomasa, 8.6\% tenían exposición por arriba de las 200 horas al año. La exposición a asbesto fue registrada en $23.4 \%(n=171)$, en la mayoría de los expuestos el grado de la exposición oscilaba alrededor de las 10 horas y la mayor exposición registrada fue de más de 40 años en 12 pacientes. La exposición a hidrocarburos se presentó en 2.52\% $(n=19)$. Se contó con pruebas de funcionamiento pulmonar en $97.7 \%$ $(n=734)$, de los cuales sólo $4.2 \%$ de los pacientes contaban con diagnóstico espirométrico de EPOC $(n=31)$. Las principales comorbilidades pulmonares registradas fueron en orden descendente: EPOC 4.2\% $(n=31)$, tuberculosis pulmonar en $2.5 \%(n=19)$, enfermedad intersticial $0.5 \%$ $(n=4)$, asma en $1 \%(n=8)$, micosis pulmonar $0.4 \%(n=$ 3) y SAOS en un paciente.

Con relación al estado funcional, la mayoría de pacientes presentó ECOG de 2, en $41.1 \%(n=309)$ al momento del diagnóstico. Los síntomas respiratorios con relación al cuadro clínico fueron reportados de la siguiente manera: tos, $87.1 \%(\mathrm{n}=654)$; disnea, $74.4 \%(\mathrm{n}=559)$; pérdida de peso, $57.12 \%(n=429)$; dolor torácico, $48.5 \%(n=364)$; fatiga, $33.9 \%(n=255)$; $y$ hemoptisis en $18.8 \%(n=141)$. Respecto al método diagnóstico para la toma de biopsia se realizaron procedimientos únicos y múltiples, en caso de que el primer procedimiento no hubiese mostrado diagnóstico histológico definitivo. Se registró necesidad de un sólo procedimiento diagnóstico en $90 \%(n=676)$. Los métodos diagnósticos realizados fueron de manera descendente: procedimientos broncoscópicos $35.1 \%(\mathrm{n}=237)$, guiados por US 19.7\% $(n=133)$, VATS $17 \%(n=115)$, EBUS $16.7 \%$ $(n=113)$, guiados por TAC 5.6\% $(n=38)$, toracotomía $5.3 \%$ $(n=36)$ y mediastinoscopia en $0.6 \%(n=4)$.

Respecto al tipo de CP, el CPCNP fue el más frecuente con $93.74 \%(n=704)$. De este grupo, la distribución histológica fue: adenocarcinoma en $86 \%(n=606)$, seguida de carcinoma epidermoide $12.6 \%(n=89)$, y células grandes en $1.4 \%(n=9)$. El CPCP representó $6.26 \%(n=47)$ de toda la cohorte (Figura 1).

Con relación al estadío clínico, las frecuencias de estadíos se distribuyeron de la siguiente manera: para CPCNP: ECI 4.1\% ( $n=29)$, ECII 5.4\% ( $n=38)$, ECIII 22\% $(n=155)$ y ECIV $68.5 \%(n=482)$ (Figura 2); y para CPCP: enfermedad limitada $(n=12)$ y extensa $(n=35)$. La mayoría de los pacientes en estadío III eran del grupo de enfermedad avanzada.

Al momento del diagnóstico, la enfermedad metastásica extrapulmonar se encontró en $52.8 \%(n=320)$. El sitio de

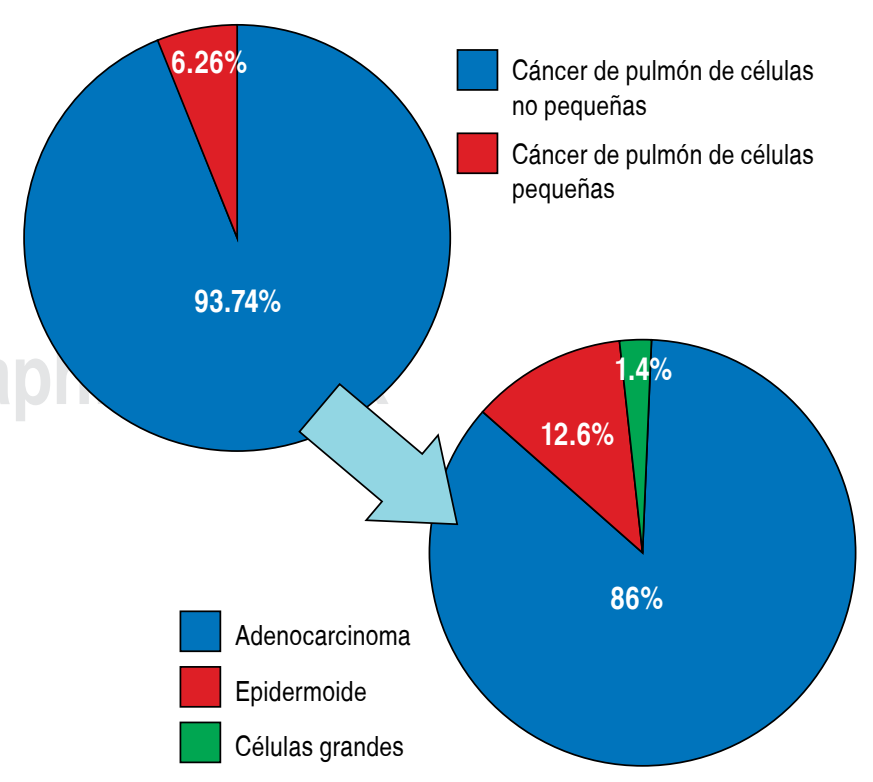

Figura 1: Epidemiología del cáncer de pulmón en el INER. 


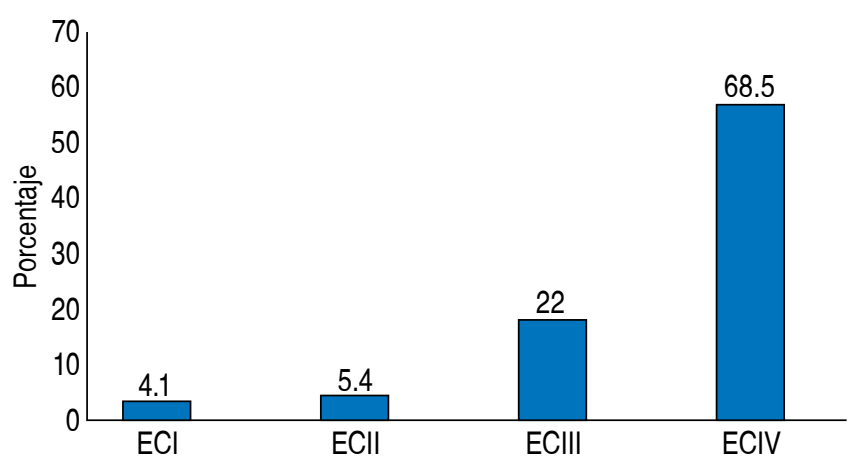

Figura 2: Etapas clínicas de cáncer pulmonar de células no pequeñas al momento del diagnóstico, INER.

metástasis extrapulmonar más frecuente fue hueso $38.6 \%$ $(n=234)$, seguido de SNC $11.7 \%(n=71)$. Se determinó el panel molecular para EGFR a un total de 460 muestras, $71.28 \%$ ( $n=432)$ de histología de adenocarcinoma y a $31.5 \%$ ( $n=28)$ de histología escamosa. Se encontró 38.19\% con resultado de mutación de sensibilidad en EGFR $(\mathrm{n}=$ 165). De estas determinaciones, los exones con mutaciones sensibles con más frecuencia registrados fueron: exón 19, seguido del exón 21, tal como se menciona en la literatura con $66.7 \%(n=110)$ y $29.1 \%(n=48)$. Los exones 18 y 20 son los menos frecuentes, encontrándose en el presente estudio en $2.4 \%(n=4)$ y $1.8 \%(n=3)$, respectivamente (Figura 3). Las mutaciones de resistencia de novo en T790M en tejido por sistema Idylla se reportaron en $8.04 \%(n=37)$.

Respecto a la correlación de positividad para mutación sensible en EGFR y antecedente exposicional se encontró asociación entre mutación negativa de EGFR y antecedente de tabaquismo con $\mathrm{p}$ estadísticamente significativa y un OR de 0.47 (IC 95\% 0.31-0.69) con valor de p de 0.0001.

El antecedente de exposición al humo de leña fue con un corte por arriba de 200 horas año en IEHL. No se encontró estadísticamente significativo OR de 0.89 (IC 95\% 0.44-1.8) con valor de p de 0.8686 para asociación con mutación en EGFR.

De las determinaciones de otras mutaciones somáticas se encontró ALK: positivo 3.3\% $(n=20)$, negativo en $25.4 \%$ $(n=154)$ y no determinado en $71.3 \%(n=432)$; para KRAS: positivo $1.5 \%(n=9)$, negativo en $26.3 \%(n=159)$ y no determinado en $72.2 \%(n=437)$; para PD-L1: positivo $4.6 \%(n=28)$, negativo en $9.7 \%(n=59)$ y no determinado en $85.7 \%(n=519)$. Del corte de PD-L1 fue: 1-10 $(n=18)$, $11-49(n=7)$ y más de $50 \%(n=3)$.

Respecto a la información de las líneas de tratamiento, hubo registro respecto a si fueron candidatos a recibir tratamiento en $95.5 \%$ de los pacientes $(n=717)$. De los tratamientos en diferentes líneas se encontró: tratamiento en primera línea recibido por $82.7 \%(n=593)$, segunda línea en $52.6 \%(n=312)$, tercera línea en $46.15 \%(n=144)$, cuarta línea en $54.16 \%(n=78)$ y más de una cuarta línea en $39.74 \%(n=31)$ de los pacientes. Del total de pacientes diagnosticados con CPCNP de estirpe adenocarcinoma candidatos a una primera línea, ésta fue recibida con esquema de quimioterapia citotóxica basada en platinos en $94 \%$ $(n=558)$. Tomando en cuenta que no hay un estándar de tratamiento en cuarta línea ni líneas subsecuentes para CP, el agente en estas líneas y subsecuentes fue considerado a discreción de los médicos oncólogos. Del total de pacientes candidatos a un tratamiento de inmunoterapia $(n=79)$, la cual se instauró en el INER vía protocolo de investigación con apoyo farmacéutico con uso de un anti-PD1, 8.9\% (n $=7)$ lo recibió en primera línea, $25.3 \%(n=20)$ en segunda línea, 43\% ( $n=34)$ en tercera línea, 19\% $(n=15)$ en cuarta línea y $3.8 \%(n=3)$ más allá de una cuarta línea.

\section{DISCUSIÓN}

El CP sigue siendo un problema de salud pública a nivel mundial y en México. El análisis epidemiológico del CP en nuestra institución reportó, al igual que la epidemiología a nivel mundial, que el tipo de CP más frecuente es el CPCNP de histología adenocarcinoma y que la etapa clínica al momento del diagnóstico sigue siendo la enfermedad avanzada. A pesar de todas las medidas implementadas en términos de prevención, el hábito tabáquico sigue siendo un problema de interés como factor de riesgo de desarrollar enfermedades crónicas y carcinogénesis a nivel pulmonar. En nuestra cohorte se encontró que a pesar de la sintomatología respiratoria manifiesta meses antes del abordaje por sospecha de CP, 31.4\% de los pacientes eran fumadores actuales al diagnóstico, valorados en su mayoría por un médico de primer nivel y/o en algunos casos por un especialista.

Si bien, el desconocimiento de la carcinogénesis por tabaco, así como la indiferencia o poca sensibilidad del

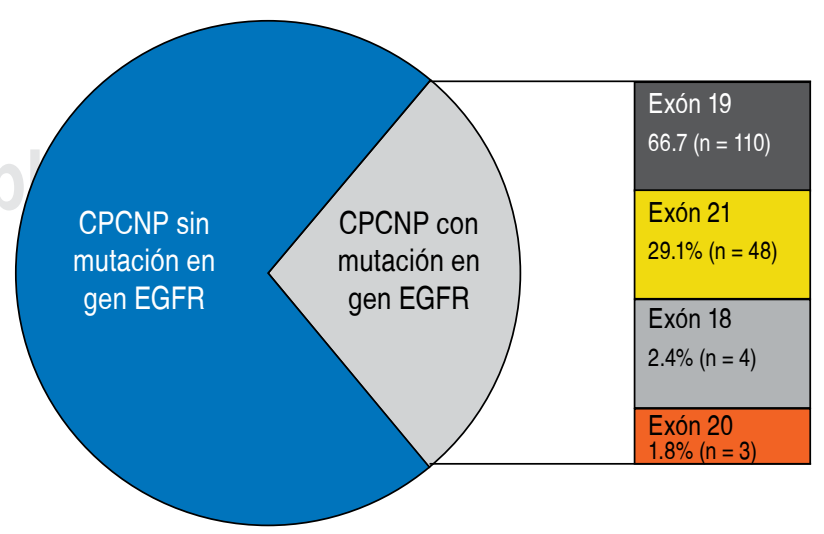

Figura 3: Cáncer de pulmón de células pequeñas y mutación sensible en el gen de EGFR. 
Neumol Cir Torax. 2019; 78 (4): 356-362

personal médico para implementaciones de suspensión de hábito tabáquico e inclusión de pacientes a un programa de tamizaje para $\mathrm{CP}$, ha contribuido a la alta incidencia de $\mathrm{CP}$ en la actualidad y al diagnóstico en etapa avanzada. La implementación de medidas antitabaco por neumólogos y médicos de primer y segundo nivel de atención puede contribuir a disminuir la mortalidad por esta enfermedad tal como lo muestran los resultados recientemente publicados del estudio NELSON. ${ }^{17}$ Por otro lado, la incidencia de $\mathrm{CP}$ en no fumadores también es un aspecto relevante. Cabe destacar que la exposición a biomasa es el segundo factor de riesgo en importancia de CP y el primero en no fumadores en países subdesarrollados, donde la combustión de leña es la principal fuente de energía. La población atendida en el INER, al ser un centro de referencia de tercer nivel para enfermedades pulmonares, muestra de manera representativa el grupo de población expuesta a biomasa a nivel nacional. Hasta el momento, sabemos que tanto un IT mayor de 20 y un IEHL mayor de 200 horas por año son factores de riesgo de desarrollar enfermedades pulmonares crónicas, específicamente patologías obstructivas como son EPOC englobando ambos fenotipos (bronquitis y enfisema), pero no así para CP. Se han realizado estudios para determinar el corte de exposición a biomasa expresado en horas/año con mayor asociación como factor de riesgo de CP sin resultados actualmente concluyentes. En el INER recién se inició un estudio retrospectivo para identificar el corte de IEHL con mayor valor de OR para CP. En nuestro estudio se encontró asociación entre mutación negativa de EGFR y antecedente de tabaquismo tal como se menciona en la literatura.

El corte de horas año en el IEHL no alcanzó significancia estadística OR de 0.89 (IC 95\% 0.44-1.8) con valor de $p$ de 0.8686 para asociación con mutación en EGFR, lo cual plantea la necesidad de estudios que identifiquen el corte de IEHL más asociado a CP y a mutación en EGFR específicamente en histología de adenocarcinoma. Respecto a otros factores de riesgo como son los hidrocarburos y el asbesto sigue existiendo dificultad para la cuantificación objetiva de la exposición, pues la metodología necesaria para realizar ensayos clínicos concluyentes no es sencilla. A pesar de esta información, estos factores son reconocidos en la actualidad por la IARC como carcinógenos, por lo que debe incluirse el antecedente exposicional en el interrogatorio médico.

En nuestra cohorte la exposición de hidrocarburos se presentó en 3\%, siendo mayor en el género masculino y en edad laboralmente productiva donde las implementaciones de mejora en materia de prevención, consideramos, podrían contribuir a disminuir la incidencia de $\mathrm{CP}$ en este grupo. Respecto a la sintomatología reportada al momento del diagnóstico encontramos, al igual que lo descrito a nivel mundial, que al inicio los síntomas son inespecíficos y que sin evidencia de imagen de masa por estudio radiográfico, la tos crónica es un síntoma por el que se inicia abordaje de patologías respiratorias diferentes a neoplasia pulmonar, retardando el diagnóstico final de cáncer. Tanto la sintomatología inespecífica como la presencia de síntomas pulmonares tardíos (por complicaciones de la enfermedad en etapa clínica avanzada) es lo que ha contribuido a que la detección del CP en la mayoría de casos se realice en etapas en las que no se puede ofrecer tratamiento curativo.

Guiber et al. realizaron en 2015 un estudio de pacientes con CP sintomáticos por obstrucción de vía aérea, en el que se les dio atención multidisciplinaria y de seguimiento de los síntomas respiratorios, y encontraron que el grupo con seguimiento neumológico clínico y de intervencionismo mostró mejor calidad de vida que el grupo sin intervención. ${ }^{18}$ A diferencia de otros centros, el INER cuenta con múltiples médicos especialistas en enfermedades pulmonares donde se ofrece a los pacientes abordajes multidisciplinarios (neumólogo clínico, neumólogo intervencionista, neumooncólogo, neumólogo especialista en terapia respiratoria, rehabilitación pulmonar, cirugía de tórax y radiólogo intervencionista, etcétera) para el diagnóstico de CP y detección temprana de comorbilidades pulmonares, las cuales al recibir tratamiento oportuno se asocian con mejora en la calidad de vida del paciente oncológico. Con relación al método diagnóstico (toma de biopsia) se registró necesidad de un solo procedimiento en $90 \%$ de los pacientes. Los métodos diagnósticos realizados fueron de manera descendente: procedimientos broncoscópicos $35.1 \%$, guiados por US $19.7 \%$, VATS $17 \%$, EBUS $16.7 \%$, guiados por TAC 5.6\%, toracotomía 5.3\% y mediastinoscopia en $0.6 \%$. En la actualidad, el instituto cuenta con infraestructura de punta necesaria para el diagnóstico personalizado de CP. En relación con la determinación de perfiles moleculares contamos con laboratorio capacitado para realización de NSG (experimental), patólogos respiratorios expertos en procesamiento de muestras de CP y manejo de Idylla (PCR automatizado para determinación de EGFR y KRAS), así como participación activa en programas de apoyo extramuros para análisis de drivers moleculares específicos (PD-L1, C-MET, BRAF, ROS-1, KRAS); todo esto con intención de ofrecer al paciente el tratamiento personalizado de mayor beneficio en control de su enfermedad.

Con respecto a la estadificación, la enfermedad metastásica extrapulmonar se encontró en $52.8 \%$, siendo el sitio de metástasis extrapulmonar más frecuente a nivel óseo seguido del SNC. Es sabido que estos datos difieren de otros centros, ya que tiene implicación estadística la metodología de estudios utilizada en cada uno. Hoy en día, el INER no cuenta con posibilidad de hacer estudios intramuros de RMN, por lo que la evaluación de enfermedad metastásica en sistema nervioso se realiza con TAC 
contrastada de cráneo cuyos valores de $S$ y E oscilan entre 90 y $70 \%$, respectivamente.

En promedio, los pacientes referidos a nuestra institución para abordaje de CP reportan una estancia hospitalaria de siete a 10 días e inicio de tratamiento de ocho a 15 días después del egreso hospitalario. La tasa de complicaciones registrada por procedimientos diagnósticos en el INER (no sólo en pacientes oncológicos) es mínima, siendo de mayor frecuencia y repercusión clínica en los pacientes ALK positivos donde el perfil clínico y tomográfico es distinto. Se realizó un estudio descriptivo en el INER donde se identificó que las complicaciones broncoscópicas al diagnóstico de estos pacientes fue de $14.2 \%$ (inherentes al procedimiento, explicados por el daño pulmonar secundario a la presentación masa-consolidación de la enfermedad). Respecto a la medicina personalizada encontramos una frecuencia de alteraciones moleculares similar a la reportada en la literatura, tal es el caso de mutación en EGFR que en nuestra población osciló en $38.19 \%$, cifra prácticamente similar a la descrita en otros estudios nacionales y de población latinoamericana. A pesar de que por cuestiones de logística algunas de las muestras no fueron evaluadas para todas las mutaciones somáticas existentes como drives moleculares, la siguiente mutación en frecuencia reportada fue el rearreglo en ALK. Tal como se mencionó con anterioridad, el perfil clínico y tomográfico de estos pacientes es diferente, incluso el perfil de respuesta a tratamientos dirigidos.

El INER tiene posibilidad de inclusión a proyectos de investigación a los pacientes con rearreglo ALK para tratamientos TKI anti-ALK vía protocolo de investigación. La enfermedad metastásica en SNC al momento del diagnóstico se encontró en 100\% de la cohorte en un estudio de caracterización de pacientes positivos en el INER, siendo sintomática sólo en un tercio de los mismos. Se sabe que la determinación molecular tiene implicaciones pronósticas con otros factores tales como son: edad, histología, ECOG y el patrón histológico. Actualmente, el servicio de patología del INER utiliza la actualización de la Organización Mundial de la Salud (OMS) 2015 que permite identificar patrones histológicos de buen pronóstico asociados a respuesta terapéutica, aquellas muestras analizadas antes de 2015 fueron reclasificadas. Se encontró, al igual que lo reportado en la literatura mundial, un predominio de patrones acinar y lepídico en 41.77 y $32.34 \%$, respectivamente. Por último, en referencia a la información de las líneas de tratamiento, encontramos que se ofreció tratamiento para CP en primera línea a $82.7 \%$, en segunda línea a $52.6 \%$, en tercera línea a $46.15 \%$, en cuarta línea $54.16 \%$ y más de una cuarta línea a $39.74 \%$ de los pacientes, lo cual traduce la cronificación de los pacientes con CP asociado, entre otros factores, a las nuevas estrategias terapéuticas y a la intervención multidisciplinaria con papel clave del médico neumólogo con entrenamiento en oncología torácica. Los esquemas preferidos en primera línea para CPCNP histología adenocarcinoma fue el esquema basado en platinos en aquellos pacientes EGFR negativos. Paclitaxel/carboplatino fue el doblete preferido por el médico oncólogo tratante salvo documentación de reacciones de hipersensibilidad al fármaco. En casos en los que la situación económica del paciente lo permitiera, se utilizaron esquemas como pemetrexed/carboplatino. Los pacientes diagnosticados con CPCNP candidatos a un TKI en primera línea de tratamiento recibieron TKI de primera o segunda generación con apoyo institucional a mujeres jóvenes con antecedente de exposición a biomasa. Gefitinib y afatinib fueron los TKI anti-EGFR más utilizados. Las toxicidades asociadas al tratamiento más frecuente reportadas fueron dolor abdominal, diarrea y rash grado 1-2 y grado 3-4 con suspensión de tratamiento excepcionalmente. Del total de la cohorte estudiada, $13 \%$ recibieron inmunoterapia con un agente anti-PD1 vía protocolo de investigación. En la actualidad tres pacientes continúan recibiendo inmunoterapia como tratamiento control del CP.

\section{CONCLUSIONES}

Sabemos que la dificultad técnica para realizar tomografías de baja dosis en contexto de screening pulmonar, la incapacidad de efectuar estudios diagnósticos mínimamente invasivos e incluso identificación de perfil molecular de $\mathrm{CP}$ que dicten el tratamiento personalizado en centros de segundo nivel y la falta de capacitación en referencia oportuna del paciente con neoplasia pulmonar a médicos de primer nivel son aspectos importantes a mejorar en nuestro país, ya que impactan en el pronóstico y calidad de vida del paciente con CP. La situación clínicoepidemiológica del INER se asemeja a las estadísticas mundiales en diagnóstico y tratamientos personalizados en contexto de CP.

\section{Limitaciones:}

En primer lugar nuestros resultados son locales, es decir, de un solo centro. Sin embargo, consideramos al INER como centro de referencia nacional que concentra gran población de pacientes con factores exposicionales relevantes para CP diferente al tabaquismo. Segundo, algunos bloques de parafina de pacientes con diagnóstico de adenocarcinoma pulmonar no fueron enviados a análisis mutacional por lo escaso de la muestra y la falta de posibilidad de rebiopsias tejido pulmonar por estado funcional o falta de autorización por parte del paciente. Tercero, no se realizó seguimiento de pacientes a largo plazo en este estudio. El patrón histológico emitido antes de la clasificación de 2015 de la OMS se reclasificó de manera cegada por el Servicio de Patología. 
Neumol Cir Torax. 2019; 78 (4): 356-362

\section{REFERENCIAS}

1. Jemal A, Siegel R, Ward E, Hao Y, Xu J, Murray T, et al. Cancer statistics, 2008. CA Cancer J Clin 2008;58(2):71-96. doi: 10.3322/ CA.2007.0010.

2. Arrieta O, Guzmán-de Alba E, Alba-López LF, et al. Consenso nacional de diagnóstico y tratamiento del cáncer de pulmón de células no pequeñas. Rev Invest Clin 2013;65(Supl. 1):s5-s84.

3. Franco-Marina F, Villalba-Caloca J. La epidemia de cáncer pulmonar en México. Rev Inst Nal Enf Resp Mex 2001;14(4):207-214.

4. Campos-Parra AD, Cruz-Rico G, Arrieta O. Genotipificación en cáncer de pulmón de células no pequeñas. GAMO 2012;11(1):35-44.

5. Peto R, Darby S, Deo H, Silcocks P, Whitley E, Doll R. Smoking, smoking cessation, and lung cancer in the UK since 1950: combination of national statistics with two case-control studies. BMJ 2000;321(7257):323-329. doi: 10.1136/bmj.321.7257.323.

6. Jemal A, Bray F, Center MM, Ferlay J, Ward E, Forman D. Global cancer statistics. CA Cancer J Clin 2011;61(2):69-90. doi: 10.3322/ caac. 20107.

7. Encuesta Nacional de Adicciones. [Fecha de acceso 12 de junio de 2019]. Accesible en: http://www.conadic.salud.gob.mx/pdfs/ena08/ ENA08_NACIONAL.pdf

8. Kerr KM. Pulmonary adenocarcinomas: classification and reporting. Histopathology 2009;54(1):12-27. doi: 10.1111/j.13652559.2008.03176.x.

9. Wallace WA. The challenge of classifying poorly differentiated tumours in the lung. Histopathology 2009;54(1):28-42. doi: 10.1111/j.13652559.2008.03181.x.

10. Arora A, Scholar EM. Role of tyrosine kinase inhibitors in cancer therapy. J Pharmacol Exp Ther 2005;315(3):971-979. doi: 10.1124/ jpet.105.084145.

11. Pérez M, Murillo R, Pinzón C, Hernández G. Costos de la atención médica del cáncer de pulmón, la EPOC y el IAM atribuibles al consumo de tabaco en Colombia (proyecto multicéntrico de la OPS). Rev Colomb Cancerol 2007;11(4):241-249.
12. Yang XD, Jia XC, Corvalan JR, Wang P, Davis CG. Development of ABX-EGF, a fully human anti-EGF receptor monoclonal antibody, for cancer therapy. Crit Rev Oncol Hematol 2001;38(1):17-23.

13. Campos-Parra AD, Cruz-Rico G, Arrieta O. Personalized treatment in non-small cell lung cancer. Rev Inv Clin 2012;64(4):377-386.

14. Wheatley-Price P, Shepherd FA. Epidermal growth factor receptor inhibitors in the treatment of lung cancer: reality and hopes. Curr Opin Oncol 2008;20(2):162-175. doi: 10.1097/ cC0.0b013e3282†335a3.

15. Herbst RS, Heymach JV, Lippman SM. Lung cancer. N Engl J Med 2008;359(13):1367-1380. doi: 10.1056/NEJMra0802714.

16. Ettinger DS, Wood DE, Aisner DL, et al. Non-small cell lung cancer, version 5.2017, NCCN Clinical Practice Guidelines in Oncology. J Natl Compr Canc Netw 2017;15(4):504-535. doi: 10.6004/ jnccn.2017.0050.

17. De Koning H, Van C, Ten K. Effects of volume CT lung cancer screening: Mortality results of the NELSON randomized-controlled population based trial. 2018 World Conference on Lung Cancer. Abstract PL02.05. Presented September 25, 2018. Chicago, Illinois, EUA.

18. Guibert N, Mazieres J, Marquette CH, Rouviere D, Didier A, Hermant C. Integration of interventional bronchoscopy in the management of lung cancer. Eur Respir Rev 2015;24(137):378-391. doi: 10.1183/16000617.00010014.

Conflicto de intereses: Dr. Jorge Arturo Alatorre Alexander: Médico de Educación Continua, Investigador y Consultor de MSD, Bristol-Myers, Roche, Takeda, Amgen, Abvie, Astra Zeneca, Boehringer Ingelheim, Pfizer, Celgen, Novartis y Bayer. Dr. Jerónimo Rodríguez Cid: Médico de educación continua, investigador y consultor de MSD, Bristol-Myers, Roche, Takeda, Amgen, Abvie, Astra Zeneca, Boehringer Ingelheim, Pfizer, Celgen, Novartis y Bayer.

Resto de investigadores no declaramos conflicto de intereses. 